\title{
All-trans retinoic acid shifts rosiglitazone-induced adipogenic differentiation to osteogenic differentiation in mouse embryonic fibroblasts
}

\author{
YING SHAO $^{1,2}$, QIAN-ZHAO CHEN ${ }^{1,2}$, YU-HUA ZENG ${ }^{1,2}$, YANG LI ${ }^{1,2}$, WEN-YAN REN ${ }^{1,2}$, LIN-YUN ZHOU $^{1,2}$, \\ RONG-XIN LIU ${ }^{1,2}$, KE WU ${ }^{1,2}$, JUN-QING YANG ${ }^{1,2}$, ZHONG-LIANG DENG $^{2,3}$, \\ YU YU ${ }^{1,2}$, WEN-JUAN SUN ${ }^{1,2}$ and BAI-CHENG HE ${ }^{1,2}$ \\ ${ }^{1}$ Department of Pharmacology, School of Pharmacy, Chongqing Medical University; ${ }^{2}$ Key Laboratory for Biochemistry \\ and Molecular Pharmacology of Chongqing; ${ }^{3}$ Department of Orthopedics, The Second Affiliated Hospital, \\ Chongqing Medical University, Chongqing, Sichuan 400016, P.R. China
}

Received April 5, 2016; Accepted October 14, 2016

DOI: $10.3892 /$ ijmm.2016.2782

\begin{abstract}
Rosiglitazone (RSG) is a potent drug used in the treatment of insulin resistance; however, it is associated with marked skeletal toxicity. RSG-induced osteoporosis may contribute to the promotion of adipogenic differentiation at the expense of osteogenic differentiation in bone marrow stromal cells. The aim of this study was to investigate whether RSG-induced bone toxicity can be reversed by combined treatment with alltrans retinoic acid (ATRA). We examined different osteogenic markers in mouse embryonic fibroblasts (MEFs) following treatment with RSG, ATRA, or RSG and ATRA in combination. We examined the effects of RSG and/or ATRA on ectopic bone formation, and dissected the possible molecular mechanisms underlying this process. We found that ATRA or RSG both induced alkaline phosphatase (ALP) activity in the MEFs, and that the ATRA-induced ALP activity was enhanced by RSG and vice versa. However, only the combination of RSG and ATRA increased the expression of osteopontin and osteocalcin, promoted matrix mineralization, and induced ectopic ossification in MEFs. Mechanistically, we found that the osteogenic differentiation induced by the combination of RSG and ATRA may be mediated partly by suppressing RSG-induced adipogenic differentiation and activating bone morphogenetic protein (BMP)/Smad signaling. On the whole, our findings demonstrate that RSG in combination with ATRA promotes the commitment of MEFs to the osteoblast lineage. Thus, the combination of these two agents may prove to be a promising
\end{abstract}

Correspondence to: Professor Bai-Cheng He or Professor Wen-Juan Sun, Department of Pharmacology, School of Pharmacy, Chongqing Medical University, 1 Yixueyuan Road, Yuzhong, Chongqing, Sichuan 400016, P.R. China

E-mail: 894704897@qq.com; hebaicheng99@yahoo.com

E-mail: 1115494605@qq.com

Key words: rosiglitazone, all-trans retinoic acid, mouse embryonic fibroblasts, osteogenic differentiation, adipogenic differentiation and novel therapeutic regimen for insulin resistance without skeletal toxicity. It may also be a better strategy with which to prevent RSG-induced osteoporosis.

\section{Introduction}

Rosiglitazone (RSG), a member of the thiazolidinedione (TZD) family, is a potent oral hypoglycemic drug used in the treatment of type 2 diabetes mellitus as an insulin sensitizer (1). However, it is associated with notorious skeletal adverse effects, such as osteoporosis, particularly in older females $(2,3)$. This has been reported due to the adipogenesis induced by RSG or its analogs, which decreases osteogenesis from bone marrow stromal cells (bMSCs) (3). Therefore, the balance between bone formation and bone absorption is distrupted. To date, bisphosphonates have been used together with RSG or its analogs in the treatment of type 2 diabetes mellitus for preventing osteoporosis by blocking osteoclastogenesis $(4,5)$. Although bisphosphonates prevent the decrease in bone density, they are also associated with some serious adverse effects (6-9). Thus, bisphosphonates may not be the best choice for the prevention of osteoporosis induced by RSG.

bMSCs are namely mesenchymal stem cells (MSCs), a type of multi-potent progenitor cells, which can differentiate into chondrogenic, adipogenic, myogenic, or osteogenic lineages $(10,11)$. RSG is an agonist of peroxisome proliferatoractivated receptor $\gamma(\operatorname{PPAR} \gamma)$, which has been considered to be a critical regulator of adipogenic differentiation $(12,13)$. PPAR $\gamma$ forms heterdimers with retinoid $\mathrm{X}$ receptor (RXR) to commit MSCs to the adipogenic lineage $(12,13)$. However, PPAR $\gamma$ activation is also essential for osteogenic differentaiton $(14,15)$. Thus, it may be possible for RSG to induce osteogenic differentiation in given conditions.

All-trans retinoic acid (ATRA), an important metabolite of vitamin A, is critical for embryonic development and maintenance in adults $(16,17)$. ATRA carries out its biological function through binding with retinoic acid receptor (RAR) or $\mathrm{RXR}$, and then forms a heterodimer with RXR to regulate the expression of downstream targets (18). Previous studies have 
indicated that ATRA can potentiate the bone morphogenetic protein (BMP)9-induced osteogenic differentiation of MSCs and pre-adipocytes $(19,20)$. Thus, ATRA may be a potential candidate to promote the osteogenic differentiation of MSCs.

In this study, we investigated whether the effects of RSG on osteogenesis are reversible. We found that the combination of RSG and ATRA induced the osteogenic differentiation of mouse embryonic fibroblasts (MEFs), which may provide a novel and promising therapeutic regimen for insulin resistance, and may promote the osteogenic differentiation of bMSCs to reverse RSG-induced osteoporosis.

\section{Materials and methods}

Cell culture and chemicals. The $\mathrm{C} 2 \mathrm{C} 12$ and $\mathrm{C} 3 \mathrm{H} 10 \mathrm{~T} 1 / 2$ cell lines were from the American Type Culture Collection (ATCC, Manassas, VA, USA). All primary antibodies were purchased from Santa Cruz Biotechnology, Inc. (Santa Cruz, CA, USA) and included anti-OPN (sc-10593), anti-OCN (sc-18319), anti-Runx2 (sc-12488), anti-Smad1/5/8 (sc-6031-R), anti-p-Smad1/5/8 (sc-12353), anti-GAPDH (sc-32233), and anti-C/EBP $\alpha$ (sc-61). ATRA was kindly provided by Chongqing Huabang Pharm aceutical Co., Ltd. (Chongqing, China). RSG was obtained from Sigma-Aldrich (St. Louis, MO, USA). The cells were maintained in Dulbecco's modified Eagle's medium (DMEM) supplemented with $10 \%$ fetal bovine serum (FBS), $100 \mathrm{U} / \mathrm{ml}$ of penicillin and $100 \mu \mathrm{g} / \mathrm{ml}$ of streptomycin at $37^{\circ} \mathrm{C}$ in $5 \% \mathrm{CO}_{2}$.

Isolation of MEFs. MEFs were isolated from NIH mice ( $\mathrm{n}=5$, obtained from The Animal Center of Chongqing Medical University; Chongqing, China) at post-coitus day 12.5, as previously described $(21,22)$. Each embryo, voided of its internal organs, was dissected and digested with $1 \mathrm{ml}$ of $0.25 \%$ trypsin at $37^{\circ} \mathrm{C}$ for $15 \mathrm{~min}$. Subsequently, $10 \mathrm{ml}$ complete DMEM were added to inactivate the trypsin. Finally, the cells were plated into $100-\mathrm{mm}$ dishes and incubated for $24 \mathrm{~h}$ at $37^{\circ} \mathrm{C}$. The non-adherent cells were discarded when the medium was changed, and the adherent cells were used as MEFs. All MEFs used in this study were at passage 5 .

RNA extraction, cDNA preparation and polymerase chain reaction. Total RNA was isolated from the cells $(\mathrm{C} 2 \mathrm{C} 12$ and C3H10T1/2 and MEFs) using TRIzol reagent (Invitrogen, Carlsbad, CA, USA) and used to generate cDNA by a reverse transcription (RT) reaction. The cDNA were further diluted with water up to 10-fold, and used for quantitative polymerase chain reaction (qPCR) or regular polymerase chain reaction (PCR) assay as templates. For regular PCR, the cycling conditions are as follows: $96^{\circ} \mathrm{C}, 45 \mathrm{sec} ; 92^{\circ} \mathrm{C}, 20 \mathrm{sec} ; 55^{\circ} \mathrm{C}, 20 \mathrm{sec} ; 72^{\circ} \mathrm{C}$, $20 \mathrm{sec} ; 4^{\circ} \mathrm{C}$, forever; the cycle number is 25 . For qPCR, the cycling conditions are as follows: $96^{\circ} \mathrm{C}, 120 \mathrm{sec} ; 95^{\circ} \mathrm{C}, 10 \mathrm{sec}$; $58^{\circ} \mathrm{C}, 20 \mathrm{sec} ; 72^{\circ} \mathrm{C}, 20 \mathrm{sec}$; the cycle number was 40 . For regular PCR, all samples were normalized to the expression level of glyceraldehyde phosphate dehydrogenase (GAPDH). The primers used in this study are listed in Table I. The results were obtained from at least 3 independent experiments.

Western blot analysis. The cells were seeded in 6-well plates and treated with ATRA, RSG, ATRA and RSG, or the same volume of DMSO as solvent control, and the concentration of ATRA and
RSG is $0.4 \mu \mathrm{M}$ and $20 \mu \mathrm{M}$, respectively. For the assay of OPN and OCN , cells were treated for 7 and 9 days. For the assay of $\mathrm{C} / \mathrm{EBP} \alpha$ and Runx 2 , cells were treated for 1 and 3 days. For the assay of Smad1/5/8 and p-Smad1/5/8, cells were treated for 1 day. At the indicated time points, the cells were lysed and the lysate was denatured by boiling for $10 \mathrm{~min}$. Total protein were separated by sodium dodecyl sulfate-polyacrylamide gel electrophoresis (SDS-PAGE), transfered onto polyvinylidene difluoride (PVDF) membranes, blocked in 10\% skimmed milk, and probed with primary antibody against the target proteins and corresponding HRP-conjugated secondary antibodies [anti-goat (SA00001-3), anti-mouse (SA00001-1), anti-rabbit (SA00001-2); Proteintech, Wuhan, China]. Finally, the target bands were visualized with SuperSignal West Pico Chemiluminescent Substrate (Thermo Scientific, Waltham, MA, USA). The results were obtained from at least 3 independent experiments.

Alkaline phosphatase (ALP) activity assay. ALP activity was determined using a modified Great Escape SEAP Chemiluminescence assay (BD Biosciences Clontech, San Jose, CA, USA) and/or histochemical staining as previously described $(14,21,22)$. The cells (MEFs) were treated with ATRA, RSG, ATRA and RSG, or the same volume of DMSO as solvent control for 5 and 7 days, and the concentration of ATRA and RSG is $0.4 \mu \mathrm{M}$ and $20 \mu \mathrm{M}$, respectively. ALP activity was measured on days 5 and 7 following treatment. For the chemiluminescence assay, each assay was performed in triplicate, and the ALP activity was normalized with the total cellular protein. All results were obtained from at least 3 independent experiments.

Oil Red $O$ staining. The cells (MEFs) were seeded in a 24-well plate, and treated with ATRA, RSG, ATRA and RSG, or the same volume DMSO as solvent control for 14 days, and the concentration of ATRA and RSG is $0.4 \mu \mathrm{M}$ and $20 \mu \mathrm{M}$, respectively. On day 14 after treatment, the cells were fixed with $10 \%$ formalin for $30 \mathrm{~min}$ and stained with Oil Red O solution (Solarbio Science \& Technology, Beijing, China). Images were taken under a microscope (Eclipse Ti-S; Nikon, Japan). For quantification, the Oil Red $\mathrm{O}$ in the cells was extracted using isopropanol, and the adsorption was measured at $570 \mathrm{~nm}$ using a spectrophotometer (ELx800; BioTek, Winooski, VT, USA). All results were obtained from at least 3 independent experiments.

Matrix mineralization assay (Alizarin Red S staining). The cells (MEFs) were seeded in 24-well culture plates and treated with dimethyl sulfoxide (DMSO), RSG and/or ATRA. The concentration of ATRA and RSG is $0.4 \mu \mathrm{M}$ and $20 \mu \mathrm{M}$, respectively. On day 20 after treatment, the mineralized matrix nodules were stained by means of Alizarin Red $\mathrm{S}$ staining assay $(14,21,22)$. The cells were fixed with $2.5 \%(\mathrm{v} / \mathrm{v})$ glutaraldehyde at room temperature for $10 \mathrm{~min}$. After being washed with distilled water, the cells were incubated with 2\% Alizarin Red S (Sigma-Aldrich) for 10-20 min, followed by removing the Alizarin Red $\mathrm{S}$ staining solution and rinsing the cells with phosphate-buffered saline (PBS) 1-2 times. The results were obtained from at least 3 independent experiments.

Transfection and luciferase reporter assay. The cells (MEFs) were seeded in T25 flasks and transfected with $2 \mu \mathrm{g}$ BMPR Smad binding elements luciferase reporter (p12xSBE-Luc) 
Table I. Primers used for PCR.

\begin{tabular}{lll}
\hline Gene & Primer & \multicolumn{1}{c}{ Sequence $\left(5^{\prime} \rightarrow 3^{\prime}\right)$} \\
\hline$P P A R \gamma$ & Forward & TTTTCAAGGGTGCCAGTTTC \\
& Reverse & AATCCTTGGCCCTCTGAGAT \\
$R X R \alpha$ & Forward & ACCCAGTTAGGGTGGGAATC \\
& Reverse & AACTGGGGGACATGACAGAG \\
$R X R \beta$ & Forward & GGCAACACTTAGCAGGGTTC \\
& Reverse & GCCAAATGAGAAGGAAGCAG \\
$R X R \gamma$ & Forward & TGTGGTCAACAGTGTCAGCA \\
& Reverse & AGAAGCCTTTGCAACCTTCA \\
$R A R \alpha$ & Forward & TCTCCCTGGACATTGACCTC \\
& Reverse & GTGTCTTGCTCAGGCGTGTA \\
$R A R \beta$ & Forward & AATGCCACCTCTCATTCAGG \\
& Reverse & GAATGTCTGCAACAGCTGGA \\
$R A R \gamma$ & Forward & AGGCAGCAGACTGACCATTT \\
& Reverse & TTCTGGTAGGTGTGCAGCAG \\
GAPDH & Forward & ACCCAGAAGACTGTGGATGG \\
& Reverse & CACATTGGGGGTAGGAACAC \\
Smad6 & Forward & GTGTTGCAACCCCTACCACT \\
& Reverse & GACATGCTGGCATCTGAGAA \\
Smad7 & Forward & GCATCTTCTGTCCCTGCTTC \\
& Reverse & CCGGTCTTCCTTTCCTTTTC \\
&
\end{tabular}

PPAR $\gamma$, peroxisome proliferator-activated receptor $\gamma ; \mathrm{RXR}$, retinoid X receptor; RAR, retinoic acid receptor; GAPDH, glyceraldehyde 3-phosphate dehydrogenase.

or Runt-related transcription factor 2 (RUNX2) [also known as core-binding factor subunit alpha-1 (Cbfa1)] reporter plasmid (p6xOSE2-Luc) per flask using Lipofectamine 2000 , as previously described $(23,24)$. At 16 h following transfection, the cells were re-seeded in 24-well plates and treated with DMSO, RSG and/or ATRA. The concentration of ATRA and RSG is $0.4 \mu \mathrm{M}$ and $20 \mu \mathrm{M}$, respectively. After $24 \mathrm{~h}$, the cells were lysed and subjected to luciferase assay using the Promega Luciferase assay kit (E1500; Promega, Madison, WI, USA). Luciferase activities were normalized with the total cellular protein concentrations. Each assay was performed in triplicate.

Ectopic bone formation assay. The cells (MEFs) were pretreated with DMSO, RSG and/or ATRA. After 4 days, the cells were harvested and resuspended in cold PBS $\left(4^{\circ} \mathrm{C}\right)$ for subcutaneous

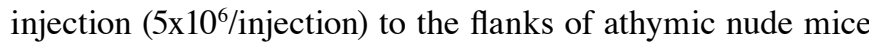
(5 animals per group, 4-6-week-old females). The animals were divided into 4 groups, including ATRA, RSG, ARTA combined with RSG, and control. RSG and/or ATRA were administrated intragastrically, 3 times a week. The same volume of carboxymethylcellulose sodium (CMC-Na) were used as a control. According to the studies and our pilot tests, the dose was set as $2.5 \mathrm{mg} / \mathrm{kg}$ for ATRA, and $20 \mathrm{mg} / \mathrm{kg}$ for rosiglitazone sodium $(2,25)$. The nude mice were obtained from the Animal Centre of Chongqing Medical University. All animal experiments were approved by the Institutional Animal Care and Use Committee (IACUC) of Chongqing Medical University. Three weeks post-implantation, the animals were euthanized with intraperitoneal injection of sodium pentobarbital $(200 \mathrm{mg} / \mathrm{kg})$, and the bone masses were retrieved for micro-computed tomographic $(\mu \mathrm{CT})$ scan and histological evaluation. The results were obtained from at least 3 independent experiments.

$\mu C T$ imaging analysis. The retrieved bone masses were scanned with $\mu \mathrm{CT}$ (VivaCT 40; Scanco Medical AG, Brüttisellen, Switzerland). The image data analysis and $3 \mathrm{D}$ reconstruction were performed following the software with the scanner $(\mu \mathrm{CT}$ 516.1).

Histological staining and evaluation. The retrieved bone masses were fixed with $10 \%$ formalin (decalcified, if necessary) and embedded in paraffin. Serial sections of the embedded specimens were stained with hematoxylin and eosin (H\&E), or Masson's trichrome (Solarbio Science \& Technology) after being deparaffinized and rehydrated.

Statistical analysis. Microsoft excel was used to calculate standard deviations and statistically significant differences between samples using the two-tailed Student's t-test. A p-value $<0.05$ was considered to indicate a statistically significant difference.

\section{Results}

$P P A R \gamma$ and isoforms of $R X R$ and $R A R$ are present in $C 2 C 12$, $C 3 H 10 T 1 / 2$ and MEFs. In this study, we aimed to ascertain whether the RSG-induced adipogenic differentiation of MSCs can be shifted towards osteogenic differentiation when used in combination with ATRA. RSG is one of the agonists of PPAR $\gamma$ and ATRA is the agonist of RAR and/or RXR. Thus, we firstly determined whether these receptors are detectable in MSCs. The results from qPCR revealed that all these receptors were present in the $\mathrm{C} 2 \mathrm{C} 12, \mathrm{C} 3 \mathrm{H} 10 \mathrm{~T} 1 / 2$ cells and MEFs, although the expression profiles differed (Fig. 1). These findings implied that RSG and ATRA can exert their biological functions through binding with corresponding receptors in these cells, respectively. The $\mathrm{C} 2 \mathrm{C} 12$ and $\mathrm{C} 3 \mathrm{H} 10 \mathrm{~T} 1 / 2$ cells are modified as cell lines for researches on oriented differentiation, such as osteogenic differentiation (26). However, MEFs are parental cells without any modifications, and can also be committed to the osteoblast lineage, at least (22). Hence, we used the MEFs in the following experiments.

RSG orland ATRA induce ALP activity in MEFs. Although it is well known that RSG is a potent inducer of adipogenesis in MSCs, our previous data (40) suggested that it may also be a potential osteogenic inducer when used in combination with ATRA. In order to clarify whether RSG can induce osteogenesis, we firstly investigated the ability of RSG or ATRA to increase ALP activity in MEFs. The results revealed that ATRA markedly increased ALP activity in the MEFs in a concentration-dependent manner, even at the concentration of $0.1 \mu \mathrm{M}$ (Fig. 2A and C). RSG also increased ALP activity in the MEFs in a concentration-dependent manner, although the required minimal concentration was much higher than that of ATRA (Fig. 2B and D). These results indicate that both ATRA and RSG have the potential to induce ALP activity in MEFs. We then wished to ascertain whether there exists a synergistic effect between RSG and ATRA in inducing ALP activity in MEFs. The 


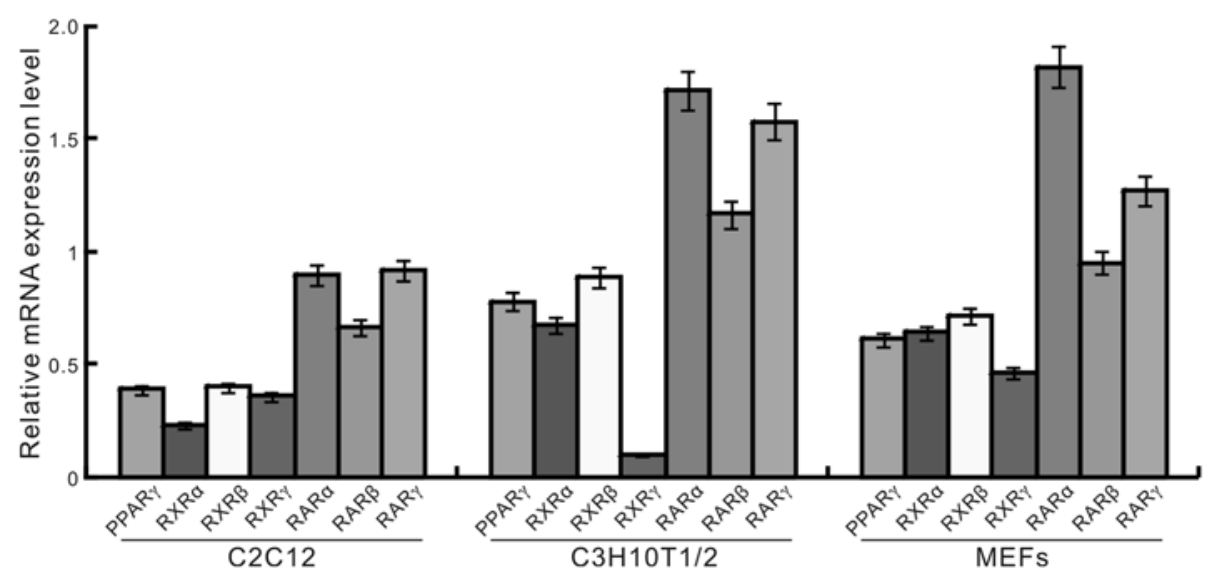

Figure 1. Endogenous expression of peroxisome proliferator-activated receptor $\gamma(\mathrm{PPAR} \gamma)$, retinoid X receptor (RXR) and retinoic acid receptor (RAR) in C2C12 and C3H10T1/2 cells and mouse embryonic fibroblasts (MEFs). qPCR of the endogenous expression of PPAR $\gamma$ and the different isoforms of RXR and RAR in the available cell lines and MEFs. The results are expressed as the ratio of mRNA expression level of each receptor to the corresponding GAPDH expression.

A

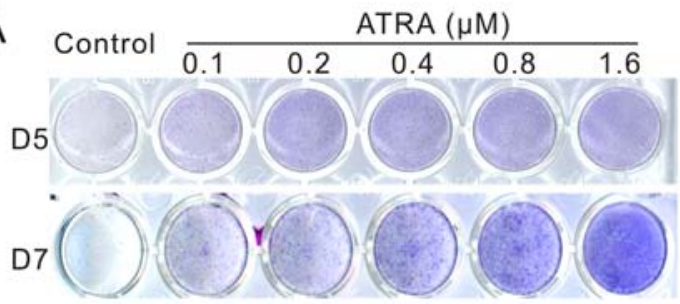

C

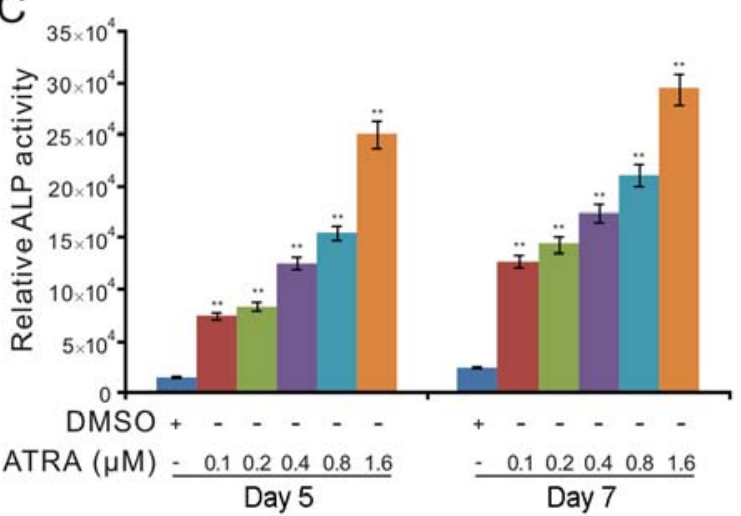

E

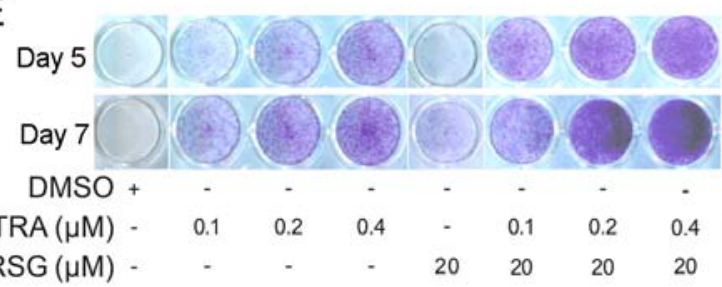

B
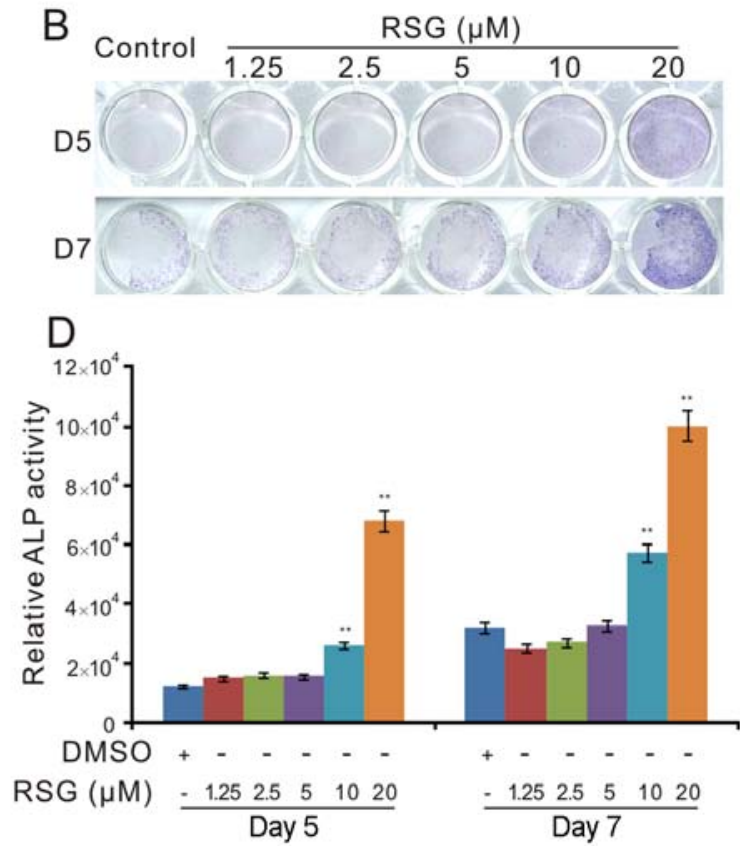

F

Day 7

DMSO +

ATRA $(\mu M)$.

RSG $(\mu \mathrm{M})$ -

Figure 2. Effects of rosiglitazone (RSG) or/and all-trans retinoic acid (ATRA) on the alkaline phosphatase (ALP) activity in mouse embryonic fibroblasts (MEFs). (A) ALP staining results show the effect of ATRA on the ALP activity in MEFs. (B) ALP staining results show the effect of RSG on the ALP activity in MEFs. (C) ALP activity assay results show the effect of ATRA on ALP activity in MEFs (** $<<0.01$ vs. control). (D) ALP activity assay results show the effect of RSG on ALP activity in MEFs $(* *$ p $<0.01$ vs. control). (E) ALP staining results show the effect of RSG on ATRA-induced ALP activity in MEFs. (F) ALP staining results show the effect of ATRA on RSG-induced ALP activity in MEFs.

results revealed that RSG substantially increased the ATRAinduced ALP activity in the MEFs (Fig. 2E), and ATRA also markedly promoted the RSG-induced ALP activity (Fig. 2F). These data suggest that the combination of ATRA and RSG induces the osteogenic differentiation of MEFs.
RSG and ATRA increase the expression of osteogenic markers and matrix mineralization in MEFs. Although RSG in combination with ATRA substantially increased ALP activity in the MEFs, this is not absolute for osteogenic differentiation. Thus, we wished to determine whether the combination of 
A
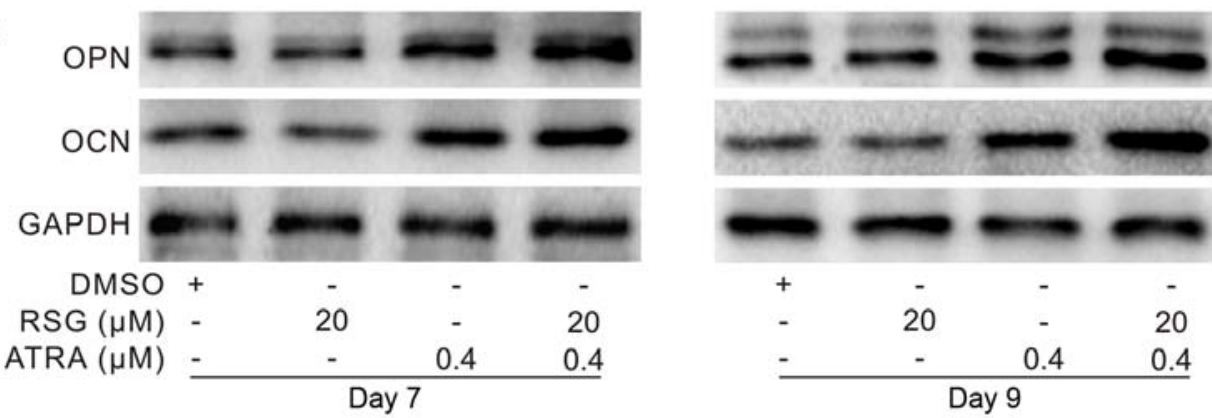

B
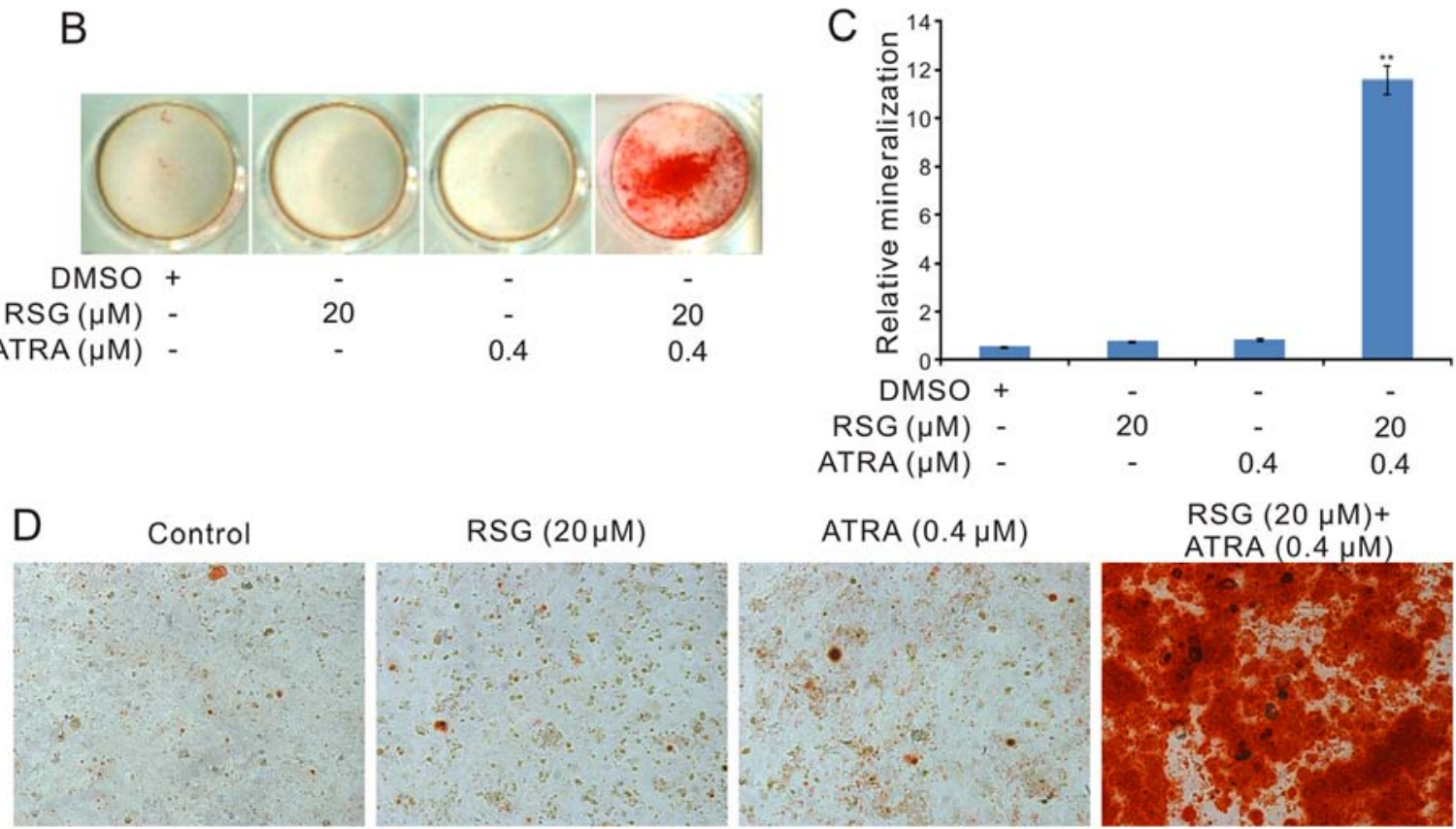

$\operatorname{RSG}(\mu \mathrm{M})$ $\operatorname{ATRA}(\mu \mathrm{M})$

0.4
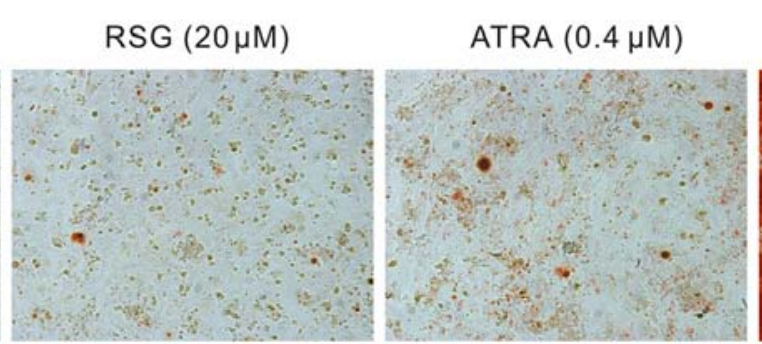

RSG $(20 \mu \mathrm{M})+$ $\operatorname{ATRA}(0.4 \mu \mathrm{M})$

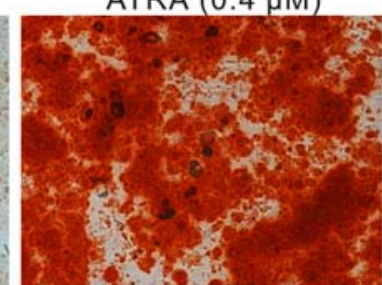

Figure 3. Effects of rosiglitazone (RSG) and/or all-trans retinoic acid (ATRA) on different osteogenic markers in mouse embryonic fibroblasts (MEFs). (A) Results of western blot analysis show the effect of RSG and/or ATRA on the expression of osteopontin (OPN) and osteocalcin (OCN) in MEFs. GAPDH was used as a loading control. (B) Alizarin Red S staining results show the effect of RSG and/or ATRA on matrix mineralization in MEFs. (C) Quantification results of Alizarin Red S staining show the effect of RSG and/or ATRA on matrix mineralization in MEFs. (D) Representative images of Alizarin Red S staining results show the effect of RSG and/or ATRA on matrix mineralization in MEFs.

RSG and ATRA induces the epxression of well-established osteogenic markers in MEFs, such as osteopontin (OPN), osteocalcin $(\mathrm{OCN})$, as well as matrix mineralization. We treated the MEFs with RSG and/or ATRA and found that the combination of RSG and ATRA not only substantially increased the expression of OPN and OCN (Fig. 3A), but it also induced matrix mineralization in the MEFs (Fig. 3B-D). However, no obvious matrix mineralization was observed when the MEFs were treated with RSG or ATRA alone (Fig. 3B-D). These results demonstrate that the combination of RSG and ATRA may be used to induce osteogenic differentiation, at least in vitro.

RSG and ATRA induce ectopic bone formation in MEFs. Although the resutls from our in vitro exeriments indicated that RSG can induce osteogenic differentiation when used in combination with ATRA, it remains unknown whether RSG and ATRA can induce bone formation in vivo. We thus performed the well-established ectopic bone formation experiment to determine the effects of RSG and/or ATRA on ossification. We pre-treated MEFs with RSG and/or ATRA, and then implanted the cells subcutaneously into the flanks of athymic nude mice, and the mice were then treated with RSG and/or ATRA intragastrically. At the end of the experiment, all nude mice were sacrificed and the bone mass was harvested; bone mass was only found in the group treated with RSG and ATRA (Fig. 4A). The 3D reconstruction of the micro-CT scan and the results of histological analysis confirmed the ossification induced by the combination of RSG and ATRA in the MEFs (Fig. 4B-D). These data strongly suggest that RSG induce sosteogenic differentiation when used in combination with ATRA in MSCs.

ATRA inhibits RSG-induced adipogenic differentiation in $M E F s$. Although the results demonstrated that RSG induced osteogenesis in MEFs when used in combination with ATRA, the possible molecular mechanisms underlying this process remain unknown. As RSG-induced osteoporosis mainly results from the commitment of bMSCs to the adipogenic lineage, we firstly analyzed whether the RSG-induced adipogenic differentiation can be inhibited by ATRA. The results of Oil Red O staining revaled that ATRA markedly suppressed RSG-induced 

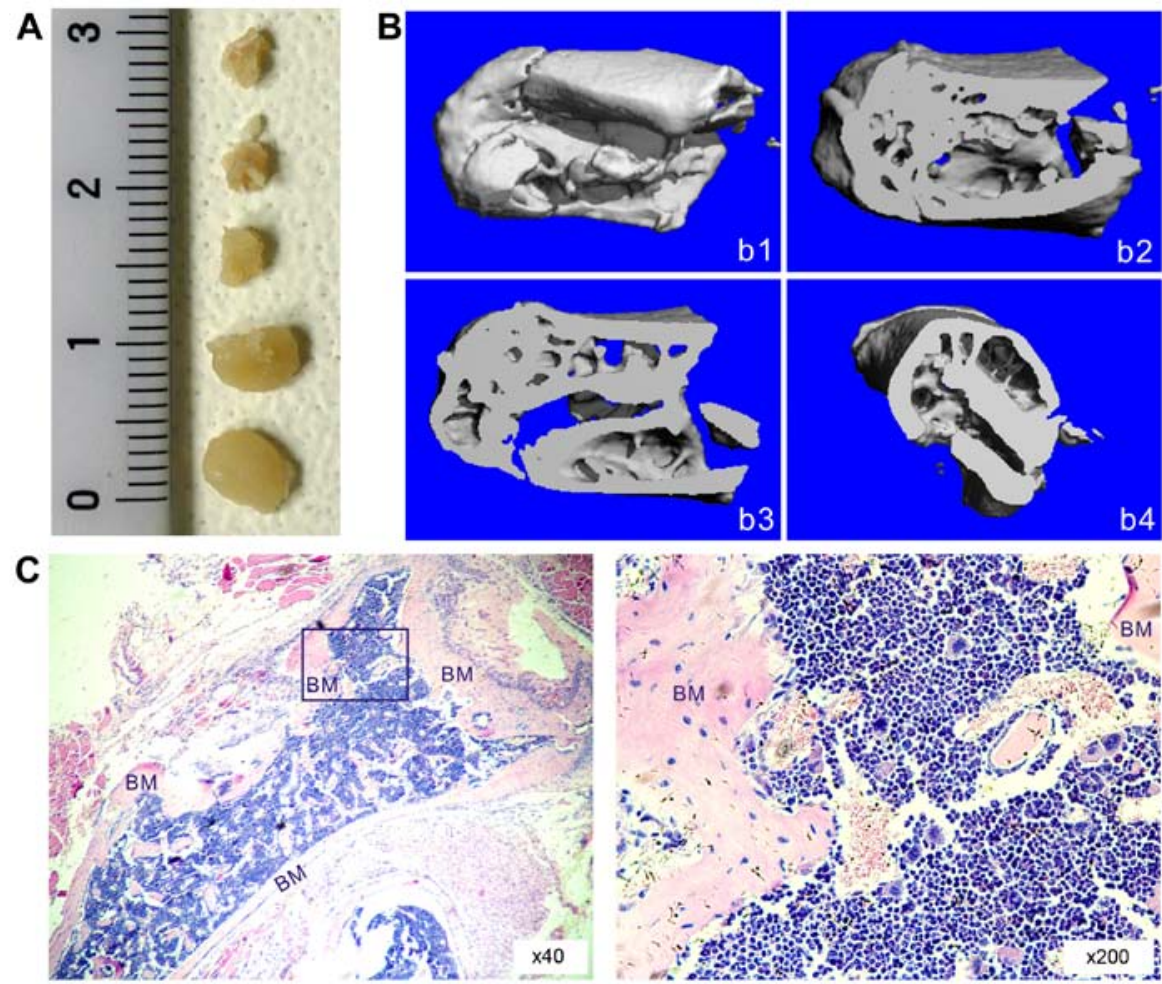

D
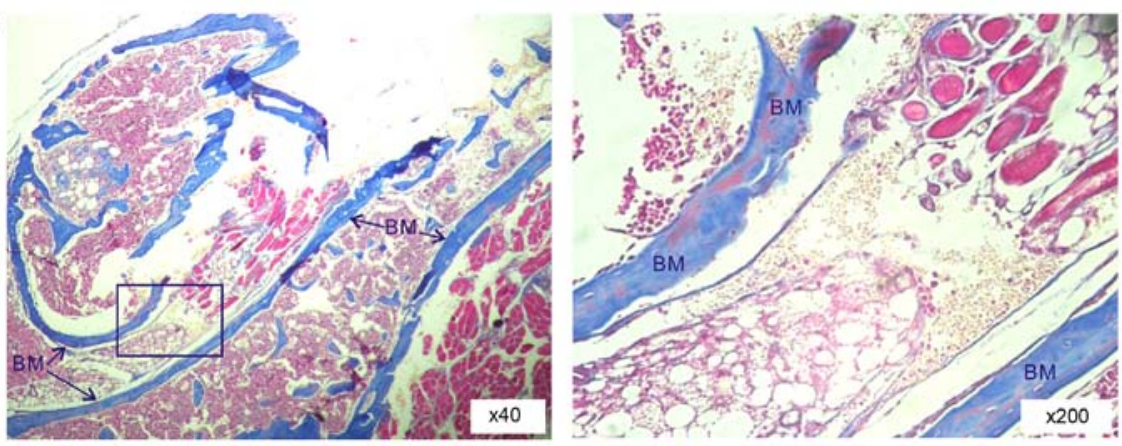

Figure 4. Effects of rosiglitazone (RSG) and/or all-trans retinoic acid (ATRA) on ectopic ossification in mouse embryonic fibroblasts (MEFs). (A) Representative images of bone mass from the ectopic bone formation induced by the combination of RSG and/or ATRA in MEFs (bone mass was only available in the group treated with RSG and ATRA). (B) Representative 3D reconstruction results of bone mass show the ectopic bone formation induced by the combination of RSG and ATRA in MEFs [(b1) general view; (b2-b4) different cross-sections of the representative bone mass]. (C) H\&E staining results show the effect of RSG and ATRA on bone formation in MEFs (image on the right panel is the magnified image of the section indicated by the rectangle on the left panel; BM, bone matrix). (D) Masson's trichrome staining results show the effect of RSG and ATRA on bone formation in MEFs (image on the right panel is the magnified image of the section indicated by the rectangle on the left panel).

adipogenesis in MEFs (Fig. 5A and B). The results of western blot analysis also revealed that ATRA markedly decreased the RSG-induced expression of CCAAT/enhancer binding protein $\alpha(\mathrm{C} / \mathrm{EBP} \alpha)$ in the MEFs (Fig. 5C and D). These data suggest that the promoting effects of the combined use of RSG and ATRA on osteogenic differentiation may partly contribute to the inhibitory effect of ATRA on the RSG-induced adipogenic differentiation of MEFs.

$R S G$ and ATRA promote BMP/Smad signal transduction in $M E F s$. Apart from inhibiting the adipogenic differentiation induced by RSG, some other important mechanisms for the osteogenesis induced by RSG and ATRA may be that osteogenic signaling or critical regulators are upregulated simultaneously. Runx 2 is one of the early critical osteogenic transcriptional factors, and we found that the transcriptional activity of Runx2 luciferase reporter was markedly increased by RSG and
ATRA (Fig. 6A), which suggested that Runx2 was upregulated. The results from western blot analysis demonstrated that RSG and ATRA increased the level of Runx2 in MEFs (Fig. 6B). Following further investigations with luciferase reporter assay and western blot analysis, we found that the transcriptional activity of the BMPR/Smad reporter was markedly increased when RSG was used in combination with ATRA in MEFs (Fig. 6C). Although there was no obvious effect on the total level of Smad1/5/8, the level of phosphorylated Smad1/5/8 (p-Smad1/5/8) was markedly increased when RSG was used in combination with ATRA (Fig. 6D). The results of PCR assay revealed that ATRA, or RSG in combination with ATRA markedly increased the expression of Smad6, but inhibited the expression of Smad7 (Fig. 6E). These data indicated that the osteogenic differentiation induced by RSG and ATRA may also be mediated by the activation of BMP/Smad signaling in MEFs. 

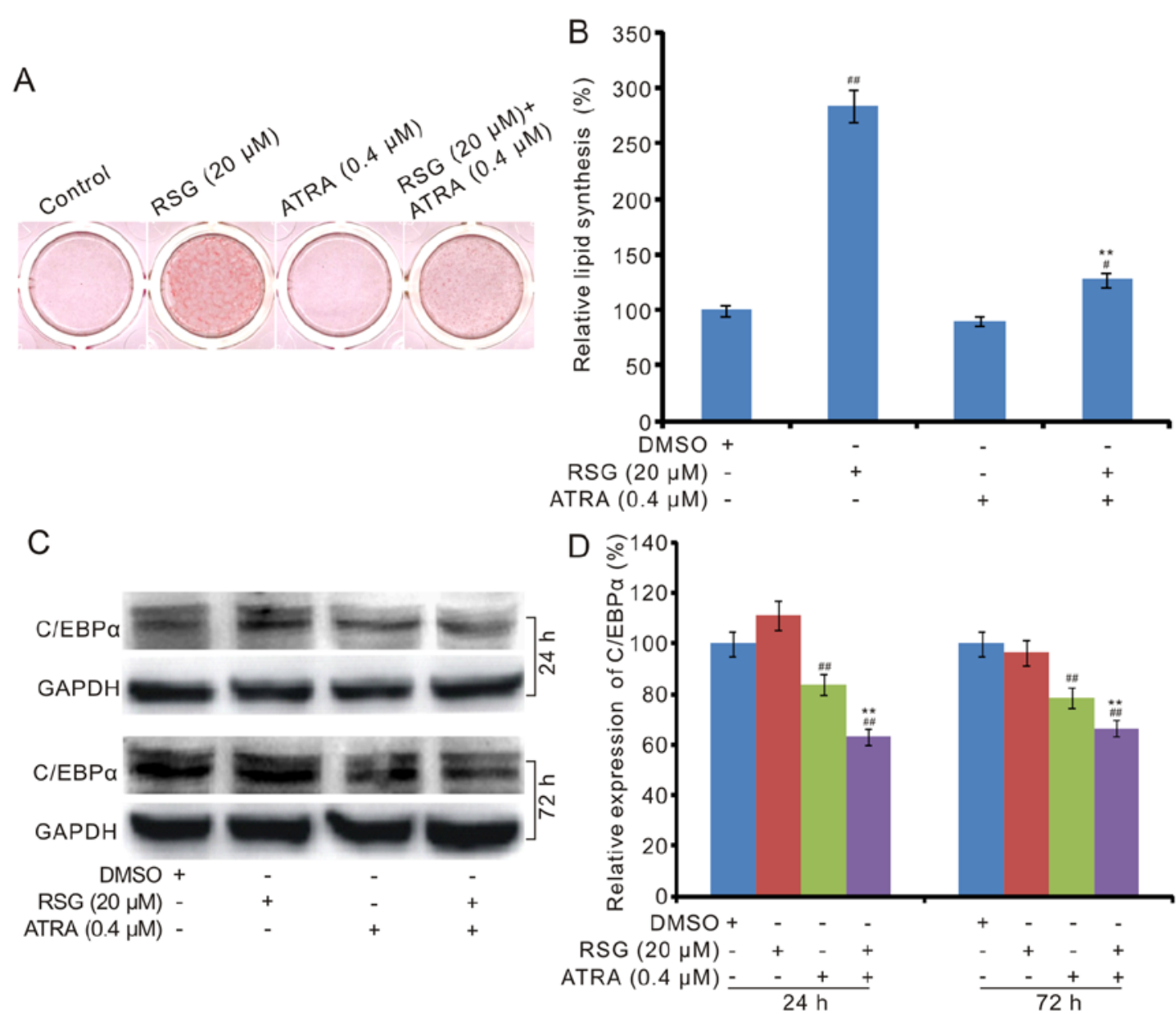

Figure 5. Effects of rosiglitazone (RSG) and/or all-trans retinoic acid (ATRA) on the adipogenic differentiation of mouse embryonic fibroblasts (MEFs). (A) Oil Red O staining results show the effect of RSG and/or ATRA on adipogenesis in MEFs. (B) Quantification of Oil Red O staining results shows the effect of RSG and/or ATRA on adipogenesis in MEFs ( $" \mathrm{p}<0.05 \mathrm{vs}$. control; "\# $\mathrm{p}<0.01 \mathrm{vs.} \mathrm{control;}{ }^{* *} \mathrm{p}<0.01 \mathrm{vs} \mathrm{RSG}$ alone). (C) Western blot analysis results show the effect of RSG and/or ATRA on the expression of C/EBP $\alpha$ in MEFs. GAPDH was used as a loading control. (D) Quantification of western blot analysis results shows the effect of RSG and/or ATRA on the expression of C/EBP $\alpha$ in MEFs (\#\# $\mathrm{p}<0.01$ vs. control; ${ }^{* *} \mathrm{p}<0.01 \mathrm{vs}$. RSG).

\section{Discussion}

In the present study, we demonstrated that RSG induced the osteogenic differentiation of MEFs when used in combination with ATRA. Mechanistically, we found that this effect may be mediated partly by the inhibition of adipogenic differentiation and the activation of BMP/Smad signaling. Our findings suggested that the combination of RSG and ATRA may be a novel and promising therapeutic regimen for type 2 diabetes mellitus, which may be used to prevent the development of osteoporosis from RSG or its analogs, although further studies are warranted to provide more evidence of this.

As RSG is a well-known agonist of PPAR $\gamma$ and ATRA is an agonist of RAR and RXR, RSG and/or ATRA may exert their biological functions though binding with the corresponding receptors $(27,28)$. For this reason, we firstly determined whether these receptors are detectable in available cells. With qPCR assay, we found that PPAR $\gamma$ and the isoforms of RAR and RXR were all present in the $\mathrm{C} 2 \mathrm{C} 12, \mathrm{C} 3 \mathrm{H} 10 \mathrm{~T} 1 / 2$ cells and MEFs, although the expression profiles differed (Fig. 1). This evidence suggested that the osteogenic differentiation induced by RSG in combination with ATRA may be initialized by binding with these receptors. As has been reported, $\mathrm{C} 2 \mathrm{C} 12$ and $\mathrm{C} 3 \mathrm{H} 10 \mathrm{~T} 1 / 2$ cells are both widely used in research on oriented differentiation (26), and MEFs are also multiple progenitor cells and can be committed to different lineages, such as osteogenic differentiation (22). In fact, $\mathrm{C} 2 \mathrm{C} 12$ and $\mathrm{C} 3 \mathrm{H} 10 \mathrm{~T} 1 / 2$ are commercially available cell lines which have been modified, while MEFs are parental cells without any modifications. In view of this, we employed the parental MEFs in our experiments.

RSG, or its analogs, have been used as powerful insulin sensitizers for type 2 diabetes mellitus, although several severe adverse effects have been reported with their use. Osteoporosis or osteoporosis-related fractures are one of these well-documented adverse effects of RSG, particularly in older females $(2,29,30)$. The hypoglycemic effect of RSG or its analogs mainly contributes to the activation of PPAR $\gamma$, a nuclear receptor regulating glucose and fatty acid metabolism. PPAR $\gamma$ needs to form a heterodimer with RXR, and then binds with PPAR responding element (PPRE) to regulate the expression of target genes. Apart from this, PPAR $\gamma$ has also been thought as an essential factor to induce adipogenic differentiation (12). bMSCs are multipotent progenitor cells, which can differentiate into the adipogenic or osteogenic lineage. Therefore, osteoporosis induced by RSG or its analogs is considered to occur by promoting bMSCs differentiate into adipocytes at the expense of osteogenesis, 

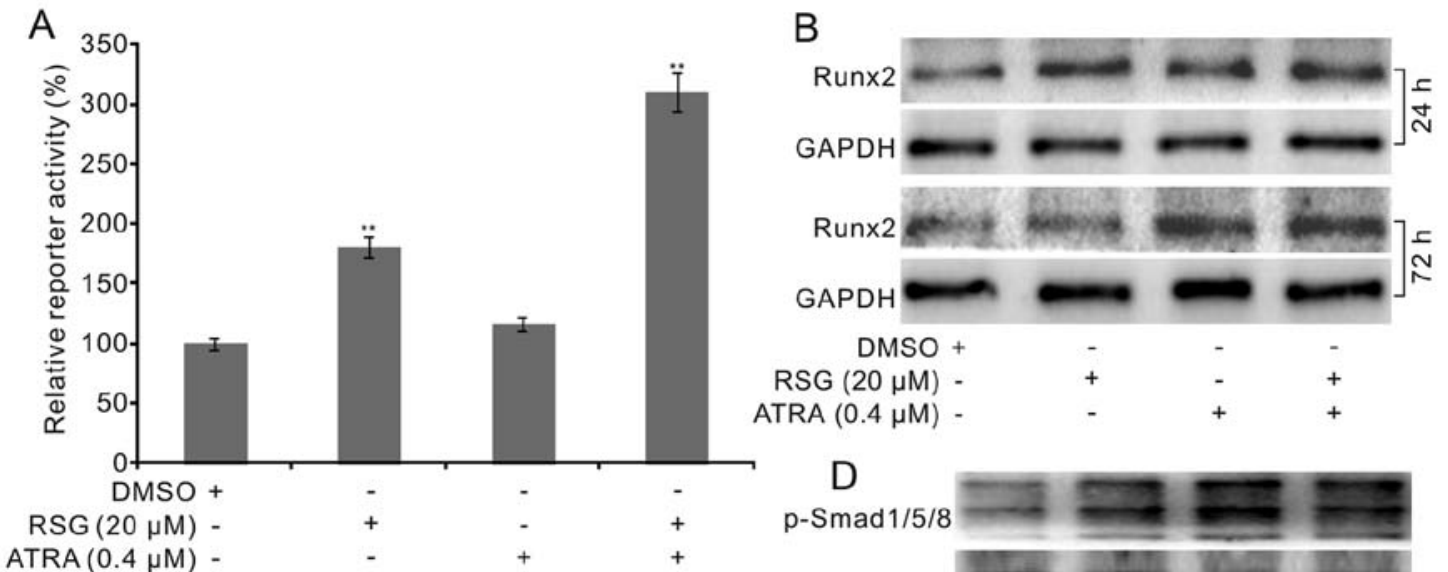
$\operatorname{ATRA}(0.4 \mu \mathrm{M})$

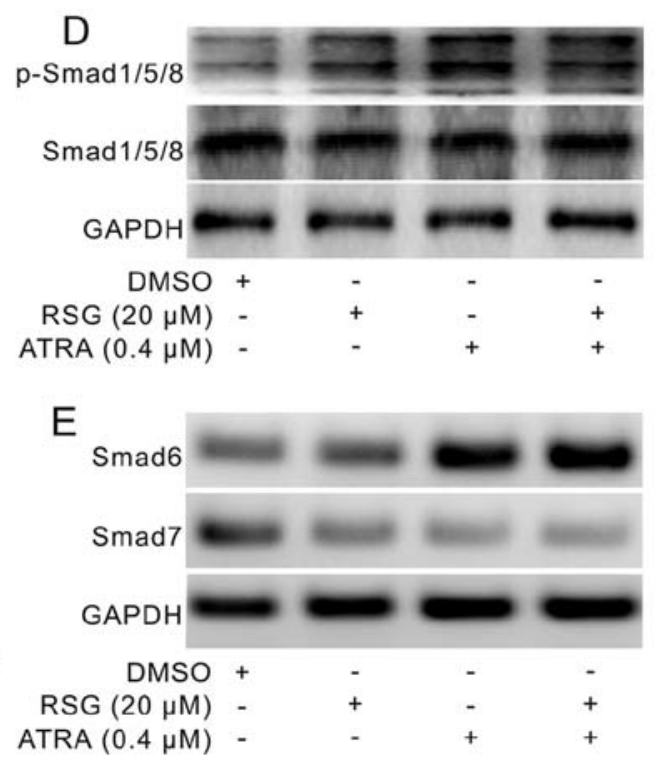

Figure 6. Effects of rosiglitazone (RSG) and/or all-trans retinoic acid (ATRA) on the expression of Runx2 and bone morphogenetic protein (BMP)/Smad signal transduction in mouse embryonic fibroblasts (MEFs). (A) Luciferase reporter assay results show the effect of RSG and/or ATRA on the transcriptional activities of p6xOSE-Luc reporter ( ${ }^{* *} \mathrm{p}<0.01$ vs. control). (B) Western blot analysis results show the effect of RSG and/or ATRA on the expression of Runx2 in MEFs. GAPDH was used as a loading control. (C) BMPR Smad binding element luciferase reporter (p12xSBE-Luc) assay results show the effect of RSG and/or ATRA on the activation of BMP/Smad signal transduction in MEFs ( $\mathrm{p}<0.05$ vs. control; * $\mathrm{p}<0.01$ vs. control). (D) Western blot analysis results show the effect of RSG and/or ATRA on the level of Smad1/5/8 and p-Smad1/5/8 in MEFs. GAPDH was used as a loading control. (E) PCR assay results show the effect of RSG and/or ATRA on the expression of Smad6 and Smad7.

which disrupts the balance between bone absorption and formation (27). As the availability of potent drugs for insulin resistance remains very limited, the FDA has approved the use of RSG at the risk of potential heart toxicity. Clinically, some specific drugs are used in combination with RSG to attenuate or prevent the RSG-induced osteoporosis, such as alendronate (4), a type of bisphosphonate. Bisphosphonates show high a affinity with the bone matrix and inhibit osteoclastogenesis, which blocks the RSG-induced loss of bone mass (4). Although bisphosphonates can inhibit RSG-induced osteoporosis, they do not exert any inhibitory effects on the RSG-induced adipogenic differentiation of bMSCs. Furthermore, bisphosphonates are also associated with certain adverse effects, such as upper gastrointestinal adverse effects, osteonecrosis of the jaw and atrial fibrillation (6-9). Therefore, bisphosphonates may not be the best candidate for preventing RSG-induced osteoporosis.

Although PPAR $\gamma$ has been regarded as a critical adipogenic regulator, it is also essential for osteogenic differentiation (14). MSCs may share a few regulators, such as PPAR $\gamma$, for osteogenesis and/or adipogenesis at the early stage of differentiation and then diverge at the interference of some crucial regulators, such as C/EBP- $\alpha$ for adipogenesis and Runx 2 for osteogenesis.
BMPs are members of the transforming growth factor (TGF)- $\beta$ superfamily, and some of them are potent for the commitment of MSCs toward the osteoblast lineage, such as BMP2, BMP7 and BMP9 (31). BMP9-induced osteogenic differentiation can be greatly reduced with the knockdown of PPAR $\gamma$, but can be enhanced by the exogenous expression of PPAR $\gamma$ in MSCs (14). Our results indicated that RSG also increased ALP activity (one of the early osteogenic markers) in MEFs when used alone (Fig. 2B and D). Therefore, the activation of PPAR $\gamma$ may also hold the potential to induce osteogenesis in progenitor cells in given conditions.

ATRA, a derivative of vitamin A, plays an important role in embryonic development and the maintenance of some vital organs in adults $(28,32)$. ATRA carries out its biological function by binding with RAR or RXR and forms a dimer with RXR. The complex then binds with the responsive element to regulate the expression of downstream target genes. To date, ATRA has only been approved for the treatment of acute promyelocytic leukaemia (APL) and acne due to severe adverse effect, such as retinoic acid syndrome, which may be reduced by changing the formulation and dose (33). As ATRA and RSG both need to bind with RXR to carry out their biological functions, these two 
drugs may crosstalk on regulating a few specific physiological processes, such as insulin resistance. It has been reported that ATRA suppressess obesity and increases insulin sensitivity, and reduces the expression of resistin (34-37), and also induces embryonic stem cell differentiation into pancreatic $\beta$ cells $(38,39)$. Therefore, ATRA may also be an excellent potential candidate drug for combatting insulin resistance, and the combination of RSG and ATRA may be a possible powerful regimen for type 2 diabetes mellitus which may thus decrease the dose of RSG used and may attenuate the RSG-related adverse effects.

Apart from insulin resistance, the combination of RSG and ATRA may also possess the ability to induce the osteogenic differentiation of MSCs. Our previous study demonstrated that troglitazone (Tro), which has been removed from the market for its lethal hepatoxicity, inhibits the proliferation of osteosarcoma (OS) cells, which can be synergistically enhanced when combined with ATRA (40). Mechanstically, we found that Tro promoted the expression of some essential osteogenic markers when used in combination with ATRA, such as ALP and OPN in OS cells and MEFs (40). This finding suggested that the combination of Tro and ATRA may have the potential to induce the osteogenesis of MSCs. As one of the analogs of Tro, RSG may also share the property of Tro to induce osteogenic differentiation when combined with ATRA in MSCs. In addition, ATRA can also enhance the BMP9-induced osteogenic differentiation of MSCs or pre-adipocytes by enhancing the activation of BMP/Smad signaling, or inhibiting adipogenic differentiation $(19,20)$. However, the effects of ATRA on osteogenic differentiation remain controversial. A few studies have indicated that ATRA can inhibit osteogenic differentiation $(41,42)$ and high doses of ATRA can induce osteoporosis (25). The differential effects of ATRA on osteogenic differentiation may depend on the cell types and concentrations used, and the concentration or dose of ATRA may be the critical factor to determine the outcome, namely osteogenesis or osteoporosis. Therefore, we hypothesized that the combination of RSG and ATRA may induce the osteogenic differentiation of MSCs under given conditions.

In this study, we found that both RSG and ATRA induced ALP activity in MEFs. RSG synergistically increased the ALP activity induced by ATRA (Fig. 2), but only the combination of RSG and ATRA markedly increased the expression of OPN and OCN, promoted matrix mineralization (Fig. 3) and induced ectopic bone formation (Fig. 4). These results were also recaptured in $\mathrm{C} 3 \mathrm{H} 10 \mathrm{~T} 1 / 2$ and $\mathrm{C} 2 \mathrm{C} 12$ cells (data not shown). Furthermore, the combination of RSG and ATRA exhibited a stronger potential to enhance BMP9 induced ALP activity in MEFs (data not shown) than that of the traditional osteogenic cocktail, which includes $50 \mu \mathrm{M}$ ascorbic acid, $10 \mathrm{mM} \beta$-glycerophosphate and $10 \mathrm{nM}$ dexamethasone (43). This evidence also suggests that the combination of RSG and ATRA can promote the commitment of progenitor cells to osteogenic differentiation.

Several physiological processes are involved in the regulation of bone development, such as adipogenesis, bone absorption and bone formation. $\mathrm{C} / \mathrm{EBP} \alpha$ is one of the essential regulators of adipogenic differentiation. BMP/Smad signaling is one of the major signaling pathways regulating bone formation, which is mediated by the phosphorylation of Smad1/5/8 (p-Smad1/5/8) and is blocked by Smad6. Our data demonstrated that the combi- nation of RSG and ATRA induced osteogenic differentiation and that this may be mediated by the inhibition of RSG-induced adipogenic differentiation (Fig. 5), and the activation of BMP/ Smad signaling transduction (Fig. 6).

Taken together, our data demonstrated that the combination of RSG and ATRA can potently induce the osteogenic differentiation of MEFs, which may be partly mediated by the inhibition of adipogenic differentiation and the activation of BMP/Smad signaling. This finding may provide a promising and innovational therapeutic regimen for insulin resistance with the combination of RSG and ATRA, which may reduce or eliminate the skeletal adverse effects of RSG by shifting the RSG-induced adipogenic differentiation towards the osteogenic differentiation of bMSCs.

\section{Acknowledgements}

We would like to thank Dr Di Chen of the University of Rochester Medical Center for providing the BMPR Smad binding elements reporter plasmids (p12xSBE-Luc). This study was supported by the research grants from the National Natural Science Foundation of China (NSFC, 81372120 and 81572226 to B.-C.H.).

\section{References}

1. Tiikkainen M, Häkkinen AM, Korsheninnikova E, Nyman T, Mäkimattila $S$ and Yki-Järvinen $\mathrm{H}$ : Effects of rosiglitazone and metformin on liver fat content, hepatic insulin resistance, insulin clearance, and gene expression in adipose tissue in patients with type 2 diabetes. Diabetes 53: 2169-2176, 2004.

2. Sardone LD, Renlund R, Willett TL, Fantus IG and Grynpas MD: Effect of rosiglitazone on bone quality in a rat model of insulin resistance and osteoporosis. Diabetes 60: 3271-3278, 2011.

3. Falchetti A, Masi L and Brandia ML: Thiazolidinediones and bone. Clin Cases Miner Bone Metab 4: 103-107, 2007.

4. Kumar S, Hoffman SJ, Samadfam R, Mansell P, Jolette J, Smith SY, Guldberg RE and Fitzpatrick LA: The effect of rosiglitazone on bone mass and fragility is reversible and can be attenuated with alendronate. J Bone Miner Res 28: 1653-1665, 2013.

5. Ettinger MP: Aging bone and osteoporosis: Strategies for preventing fractures in the elderly. Arch Intern Med 163: 2237-2246, 2003.

6. Iglesias JE, Salum FG, Figueiredo MA and Cherubini K: Important aspects concerning alendronate-related osteonecrosis of the jaws: A literature review. Gerodontology 32: 169-178, 2015.

7. Piscitelli P, Auriemma R, Neglia C and Migliore A: Alendronate: New formulations of an old and effective drug to improve adherence avoiding upper gastrointestinal side effects. Eur Rev Med Pharmacol Sci 18: 3788-3796, 2014.

8. Howard PA, Barnes BJ, Vacek JL, Chen W and Lai SM: Impact of bisphosphonates on the risk of atrial fibrillation. Am J Cardiovasc Drugs 10: 359-367, 2010.

9. Brown JP, Morin S, Leslie W, Papaioannou A, Cheung AM, Davison KS, Goltzman D, Hanley DA, Hodsman A, Josse R, et al: Bisphosphonates for treatment of osteoporosis: Expected benefits, potential harms, and drug holidays. Can Fam Physician 60: 324-333, 2014.

10. Liao HT and Chen CT: Osteogenic potential: Comparison between bone marrow and adipose-derived mesenchymal stem cells. World J Stem Cells 6: 288-295, 2014.

11. Murphy MB, Moncivais K and Caplan AI: Mesenchymal stem cells: Environmentally responsive therapeutics for regenerative medicine. Exp Mol Med 45: e54, 2013.

12. James AW: Review of signaling pathways governing MSC osteogenic and adipogenic differentiation. Scientifica (Cairo) 2013: 684736, 2013.

13. Westin S, Kurokawa R, Nolte RT, Wisely GB, McInerney EM, Rose DW, Milburn MV, Rosenfeld MG and Glass CK: Interactions controlling the assembly of nuclear-receptor heterodimers and co-activators. Nature 395: 199-202, 1998. 
14. Kang Q, Song WX, Luo Q, Tang N, Luo J, Luo X, Chen J, Bi Y, He BC, Park JK, et al: A comprehensive analysis of the dual roles of BMPs in regulating adipogenic and osteogenic differentiation of mesenchymal progenitor cells. Stem Cells Dev 18: 545-559, 2009.

15. Viccica G, Francucci CM and Marcocci C: The role of PPAR $\gamma$ for the osteoblastic differentiation. J Endocrinol Invest 33 (Suppl 7): 9-12, 2010

16. Rhinn $\mathrm{M}$ and Dollé P: Retinoic acid signalling during development. Development 139: 843-858, 2012.

17. Maden M: Retinoic acid in the development, regeneration and maintenance of the nervous system. Nat Rev Neurosci 8: 755-765, 2007.

18. Pogenberg V, Guichou JF, Vivat-Hannah V, Kammerer S, Pérez E, Germain P, de Lera AR, Gronemeyer H, Royer CA and Bourguet W: Characterization of the interaction between retinoic acid receptor/retinoid X receptor (RAR/RXR) heterodimers and transcriptional coactivators through structural and fluorescence anisotropy studies. J Biol Chem 280: 1625-1633, 2005.

19. Liu Y, Liu Y, Zhang R, Wang X, Huang F, Yan Z, Nie M, Huang J, Wang Y, Wang Y, et al: All-trans retinoic acid modulates bone morphogenic protein 9-induced osteogenesis and adipogenesis of preadipocytes through $\mathrm{BMP} / \mathrm{Smad}$ and $\mathrm{Wnt} / \beta$-catenin signaling pathways. Int J Biochem Cell Biol 47: 47-56, 2014.

20. Zhang W, Deng ZL, Chen L, Zuo GW, Luo Q, Shi Q, Zhang BQ, Wagner ER, Rastegar F, Kim SH, et al: Retinoic acids potentiate BMP9-induced osteogenic differentiation of mesenchymal progenitor cells. PLoS One 5: e11917, 2010.

21. Wang JH, Liu YZ, Yin LJ, Chen L, Huang J, Liu Y, Zhang RX, Zhou LY, Yang QJ, Luo JY, et al: BMP9 and COX-2 form an important regulatory loop in BMP9-induced osteogenic differentiation of mesenchymal stem cells. Bone 57: 311-321, 2013.

22. Huang J, Yuan SX, Wang DX, Wu QX, Wang X, Pi CJ, Zou X, Chen L, Ying LJ, Wu K, et al: The role of COX-2 in mediating the effect of PTEN on BMP9 induced osteogenic differentiation in mouse embryonic fibroblasts. Biomaterials 35: 9649-9659, 2014.

23. Niger C, Luciotti MA, Buo AM, Hebert C, Ma V and Stains JP: The regulation of runt-related transcription factor 2 by fibroblast growth factor- 2 and connexin 43 requires the inositol polyphosphate/protein kinase C $\delta$ cascade. J Bone Miner Res 28: 1468-1477, 2013.

24. Zhao M, Qiao M, Oyajobi BO, Mundy GR and Chen D: E3 ubiquitin ligase Smurf1 mediates core-binding factor alpha1/Runx2 degradation and plays a specific role in osteoblast differentiation. J Biol Chem 278: 27939-27944, 2003.

25. Hotchkiss CE, Latendresse J and Ferguson SA: Oral treatment with retinoic acid decreases bone mass in rats. Comp Med 56: 502-511, 2006.

26. Chen L, Jiang W, Huang J, He BC, Zuo GW, Zhang W, Luo Q, Shi Q, Zhang BQ, Wagner ER, et al: Insulin-like growth factor-2 (IGF-2) potentiates BMP-9-induced osteogenic differentiation and bone formation. J Bone Miner Res 25: 2447-2459, 2010.

27. Ali AA, Weinstein RS, Stewart SA, Parfitt AM, Manolagas SC and Jilka RL: Rosiglitazone causes bone loss in mice by suppressing osteoblast differentiation and bone formation. Endocrinology 146: 1226-1235, 2005.
28. Duester G: Retinoid signaling in control of progenitor cell differentiation during mouse development. Semin Cell Dev Biol 24: 694-700, 2013

29. Lecka-Czernik B: Bone loss in diabetes: Use of antidiabetic thiazolidinediones and secondary osteoporosis. Curr Osteoporos Rep 8: 178-184, 2010.

30. Aubert RE, Herrera V, Chen W, Haffner SM and Pendergrass M: Rosiglitazone and pioglitazone increase fracture risk in women and men with type 2 diabetes. Diabetes Obes Metab 12: 716-721, 2010.

31. Wang RN, Green J, Wang Z, Deng Y, Qiao M, Peabody M, Zhang Q, Ye J, Yan Z, Denduluri S, et al: Bone Morphogenetic Protein (BMP) signaling in development and human diseases. Genes Dis 1: 87-105, 2014.

32. Duester G: Retinoic acid synthesis and signaling during early organogenesis. Cell 134: 921-931, 2008.

33. Patatanian E and Thompson DF: Retinoic acid syndrome: A review. J Clin Pharm Ther 33: 331-338, 2008.

34. Berry DC and Noy N: All-trans-retinoic acid represses obesity and insulin resistance by activating both peroxisome proliferation-activated receptor beta/delta and retinoic acid receptor. Mol Cell Biol 29: 3286-3296, 2009.

35. Manolescu DC, Sima A and Bhat PV: All-trans retinoic acid lowers serum retinol-binding protein 4 concentrations and increases insulin sensitivity in diabetic mice. J Nutr 140: 311-316, 2010.

36. Dakshinamurti K: Vitamins and their derivatives in the prevention and treatment of metabolic syndrome diseases (diabetes). Can J Physiol Pharmacol 93: 355-362, 2015.

37. Felipe F, Bonet ML, Ribot J and Palou A: Modulation of resistin expression by retinoic acid and vitamin A status. Diabetes 53 882-889, 2004

38. Shi Y, Hou L, Tang F, Jiang W, Wang P, Ding M and Deng $\mathrm{H}$ Inducing embryonic stem cells to differentiate into pancreatic beta cells by a novel three-step approach with activin A and all-trans retinoic acid. Stem Cells 23: 656-662, 2005.

39. Bose B, Shenoy SP, Konda S and Wangikar P: Human embryonic stem cell differentiation into insulin secreting $\beta$-cells for diabetes. Cell Biol Int 36: 1013-1020, 2012.

40. He BC, Chen L, Zuo GW, Zhang W, Bi Y, Huang J, Wang Y, Jiang W, Luo Q, Shi Q, et al: Synergistic antitumor effect of the activated PPARgamma and retinoid receptors on human osteosarcoma. Clin Cancer Res 16: 2235-2245, 2010

41. Chen M, Huang HZ, Wang M and Wang AX: Retinoic acid inhibits osteogenic differentiation of mouse embryonic palate mesenchymal cells. Birth Defects Res A Clin Mol Teratol 88: 965-970, 2010 .

42. Wang A, Ding X, Sheng S and Yao Z: Retinoic acid inhibits osteogenic differentiation of rat bone marrow stromal cells. Biochem Biophys Res Commun 375: 435-439, 2008.

43. Liu G, Shu C, Cui L, Liu W and Cao Y: Tissue-engineered bone formation with cryopreserved human bone marrow mesenchymal stem cells. Cryobiology 56: 209-215, 2008. 